\title{
Educação em saúde no ensino de graduação em enfermagem: percepção dos acadêmicos
}

\author{
Health education in undergraduate nursing education: students' perceptions \\ Educación en salud en la enseñanza de pregrado de enfermería: percepción de los \\ estudiantes
}

Recebido: 20/07/2020

Aprovado: 06/04/2021

Publicado: 14/10/2021

\author{
Michele Carla Gonçalves ${ }^{1}$ \\ Nylze Helena Guillarducci Rocha ${ }^{2}$ \\ Maria Paula Custódio Silva ${ }^{3}$ \\ Bethania Ferreira Goulart ${ }^{4}$ \\ Jesislei Bonolo do Amaral Rocha ${ }^{5}$ \\ Divanice Contim 6
}

Trata-se de estudo qualitativo de caráter descritivo e exploratório, realizado entre novembro de 2018 e abril de 2019, em uma universidade pública federal com objetivo de identificar a percepção de graduandos em enfermagem acerca da disciplina Educação em Saúde. A coleta de dados foi realizada por meio de um instrumento semiestruturado e entrevista, e a análise pelo Discurso do Sujeito Coletivo. Participaram 35 acadêmicos do nono e décimo períodos, dos quais: $80 \%$ do sexo feminino e $20 \%$ do sexo masculino; com média de idade de 24 anos; $54,28 \%$ do décimo período e $45,72 \%$ do nono período; $77,14 \%$ solteiros. Emergiram quatro ideias centrais: Importância da disciplina de Educação em Saúde para o desenvolvimento acadêmico; Práticas da disciplina de Educação em Saúde pelo olhar dos discentes; Disciplina de Educação em Saúde como estratégia de ensino para população; e Visão crítica do aprender educação em saúde. Verificou-se que a disciplina Educação em Saúde no contexto de formação do acadêmico de enfermagem pode qualificar sua atuação, mostrando-se como ferramenta de trabalho e estratégia de promoção da saúde e prevenção de agravos.

Descritores: Educação em saúde; Educação em Enfermagem; Estudantes de Enfermagem, Educação superior; Ensino.

This is a qualitative study of a descriptive and exploratory nature, carried out between November 2018 and April 2019, in a Brazilian public university. It aimed to identify the perception of undergraduate nursing students about the Health Education discipline. Data collection was performed through a semi-structured instrument and interview, and the analysis through the Discourse of the Collective Subject. Thirty-five students from the ninth and tenth periods participated, of which: $80 \%$ were female and $20 \%$ were male; with an average age of 24 years; $54.28 \%$ of the tenth period and $45.72 \%$ of the ninth period; $77.14 \%$ were single. Four central ideas emerged: Importance of the Health Education discipline for academic development; Practices of the Health Education discipline from the perspective of students; Discipline of Health Education as a teaching strategy for the population; and Critical view of learning health education. It was found that the Health Education discipline in the context of nursing academic training can qualify their performance, showing itself as a work tool and strategy for health promotion and disease prevention.

Descriptors: Health education; Education, Nursing; Students, Nursing; Education, Higher; Teaching.

Se trata de un estudio cualitativo descriptivo y exploratorio, realizado entre noviembre de 2018 y abril de 2019 , en una universidad pública federal con el objetivo de identificar la percepción de los estudiantes de pregrado de enfermería sobre la asignatura Educación en Salud. La recogida de datos se realizó a través de un instrumento semiestructurado y de una entrevista y el análisis mediante el Discurso del Sujeto Colectivo. Participaron 35 estudiantes de los períodos noveno y décimo: $80 \%$ mujeres y 20\% hombres; edad media de 24 años; $54,28 \%$ del décimo período y $45,72 \%$ del noveno; $77,14 \%$ solteros. Surgieron cuatro ideas centrales: Importancia de la asignatura de Educación en Salud para el desarrollo académico; Prácticas de la asignatura de Educación en Salud desde la perspectiva de los estudiantes; Asignatura de Educación en Salud como estrategia de enseñanza para la población; y Visión crítica del aprendizaje de la educación en salud. Se comprobó que la asignatura Educación en Salud en el contexto de la formación de los estudiantes de enfermería puede calificar su desempeño, mostrándose como una herramienta de trabajo y estrategia de promoción de la salud y prevención de enfermedades.

Descriptores: Educación en salud, Educación en Enfermería; Estudiantes de Enfermería; Educación superior; Enseñanza.

1. Enfermeira. Uberaba, MG, Brasil. ORCID: 0000-0003-1281-6384 E-mail: michele.uftm@outlook.com

2. Enfermeira. Especialista em Enfermagem Neonatal. Mestre em Atenção à Saúde. Doutoranda em Atenção em Saúde pela Universidade Federal do Triângulo

Mineiro (UFTM). Uberaba, MG, Brasil. ORCID: 0000-0002-6615-1667 E-mail: nylze@hotmail.com

3. Enfermeira. Mestre em Atenção à Saúde. Doutoranda em Atenção pela Saúde pela UFTM, Uberaba, MG, Brasil. ORCID: 0000-0001-8694-1589

E-mail: maria_paulacs@hotmail.com

4. Enfermeira. Especialista em Saúde Pública. Mestre e Doutora em Enfermagem. Professor Adjunto do Curso de Graduação em Enfermagem (CGE) da UFTM,

Uberaba, MG, Brasil. ORCID: 0000-0003-2855-6767 E-mail: bethaniagoulart@yahoo.com.br

5. Enfermeira. Mestre em Enfermagem. Doutora em Atenção à Saúde. Professor Adjunto do CGE da UFTM, Uberaba, MG, Brasil. 0RCID: 0000-0002-0591-7972

E-mail: jesisleiamaralrocha@gmail.com

6. Enfermeira. Especialista em Saúde Pública. Especialista em Pediatria e Puericultura. Mestre e Doutora em Enfermagem. Professora Associada do CGE e do

Programa de Pós-Graduação em Atenção à Saúde (PPGAS) da UFTM, Uberaba, MG, Brasil. ORCID: 0000-0001-5213-1465 E-mail: d.contim@uol.com.br 


\section{INTRODUÇÃO}

1 educação em saúde é compreendida como um processo de construção de 1 conhecimentos em saúde, visando à apropriação da temática pela população ${ }^{1}$. Trata-se 1 de um conjunto de práticas que contribuem para o aumento da autonomia individual e coletiva de sujeitos para o debate com os profissionais e os gestores, de modo a alcançar uma atenção à saúde de acordo com as necessidades dos indivíduos e das comunidades, buscando melhoria na qualidade de vida e saúde da população ${ }^{1,2}$.

0 enfermeiro contribui para a prestação de cuidados integrais de saúde a indivíduos e coletividades por meio de atribuições de supervisão, coordenação e realização das atividades de educação em saúde ${ }^{2}$.

A assistência de enfermagem de alta qualidade depende diretamente da formação de enfermeiros e tem revelado fragilidades quanto ao ensino-aprendizagem e à construção de uma postura crítico-reflexiva diante de diversas condições de saúde. 0 desenvolvimento de competências do graduando deve estar voltado para o ensino qualificado, por meio de metodologias que se adequem a diferentes situações, tanto no contexto da educação quanto em sua atuação no campo da saúde $\mathrm{e}^{1-3}$.

No que se refere à formação de profissionais da saúde, inúmeras mudanças ocorreram, repercutindo no exercício e na prática de sua atuação, como as Diretrizes Curriculares Nacionais (DCN) que trouxeram um novo formato para o processo de ensino e aprendizagem ${ }^{2}$. A educação faz parte do cotidiano das pessoas, sendo necessária a utilização de métodos de ensino inovadores para o desenvolvimento de competências, o que requer a reformulação de práticas pedagógicas ${ }^{1}$.

No âmbito da enfermagem, a Educação em Saúde (ES) é uma das atribuições imprescindíveis no processo de cuidar da saúde dos indivíduos com vistas a promover a autonomia do cuidado por meio de diálogos dinâmicos, integrando aspectos físico/mental, ambiental e social; evidenciando, assim, sua importância na formação do graduando ${ }^{4-6}$.

A ES e suas ações estão concentradas entre sujeitos, comunidade e sociedade, por meio de cuidados com a saúde; ao mesmo tempo temática relevante enquanto conteúdo disciplinar para a formação. A despeito disto, ainda existem práticas restritas voltadas para abordagem biomédica focadas nos aspectos preventivos, evidenciando, assim, que as peculiaridades do processo educativo exigem uma capacitação específica que não é inerente à formação técnica dos profissionais de saúde ${ }^{4-7}$.

A ES é instrumento fundamental para a assistência integral e função educativa, próprias da prática de enfermagem. Por sua vez, há peculiaridades do processo educativo que exigem preparação específica na formação técnica do enfermeiro ${ }^{1-10}$, o que também pede um repensar acerca do ensino de graduação em enfermagem e de como as formas de ensino vêm respondendo ao cenário de mudanças.

As DCNs para os Cursos de Graduação em Enfermagem, publicadas pelo Conselho Nacional de Educação ${ }^{11}$, indicaram que ao término da formação, os enfermeiros apresentem competências e habilidades para atuar de acordo com a complexidade e desafios apresentados no Sistema Único de Saúde (SUS), assegurando a esse graduando a superação de desafios, estimulando práticas independentes, visando progressiva autonomia e assim, percorrer um saber demandado da estrutura econômica, política e cultural, que responda as demandas da população ${ }^{12,13}$. Assim, este estudo teve como objetivo identificar a percepção de graduandos em enfermagem acerca da disciplina Educação em Saúde. 


\section{MÉTODO}

Estudo descritivo, exploratório de natureza qualitativa ${ }^{14}$, realizado em uma instituição pública federal de ensino superior localizada no interior de Minas Gerais. Os participantes da pesquisa foram graduandos do curso de Enfermagem selecionados por amostragem aleatória estratificada.

Foram incluídos graduandos que tinham realizado a disciplina Educação em Saúde; e, estar matriculado no $9^{\circ}$ e $10^{\circ}$ semestre, isto pois, nestes períodos os acadêmicos vivenciam entre outras coisas, ações de educação em saúde.

A coleta de dados foi realizada entre os meses de novembro de 2018 e abril de 2019, desenvolvida por meio de um instrumento semiestruturado, composto em duas partes: dados sociodemográficos (sexo, idade, estado civil e período letivo) e um roteiro com questões norteadoras, a saber: Qual a sua concepção sobre Educação em Saúde? O que significou para você a experiência na disciplina de Educação em Saúde? Como você se sentiu ao realizar as práticas da disciplina de Educação em Saúde?

As entrevistas foram agendadas e gravadas em formato digital, conforme disponibilidade dos participantes em ambiente privativo. À realização das entrevistas, procedeu-se à explicação da importância da pesquisa e da leitura do Termo de Consentimento Livre e Esclarecido (TCLE). Todos os participantes assinaram-no confirmando a sua anuência.

As entrevistas tiveram duração média de 40 minutos; após, foram transcritas no editor de texto Microsoft Word. Os sujeitos foram identificados por meio da letra E seguida de numeral referente à ordem da entrevista (E1, E2, E3...E35.), para o sigilo dos participantes.

Para organização e interpretação dos dados optou-se pelo Discurso do Sujeito Coletivo (DSC), que compreende quatro figuras metodológicas: Expressões Chave (ECH), que revelam a essência do conteúdo dos depoimentos; as Ideias Centrais (IC), que indicam o sentido ou a expressão linguística de um depoimento analisado e de cada conjunto de ECH de mesmo sentido ou sentido complementar que irão compor, posteriormente, o DSC; Ancoragem (AC), que compreende pressupostos, princípios, hipóteses e teorias que dão sustentação às expressões chave e o DSC. O passo seguinte foi agrupar as ECH de todas as entrevistas referentes às IC destacadas; sendo possível, assim, formular e construir DSC redigido na primeira pessoa do singular e elaborado com os mais significativos extratos de depoimentos de sentido semelhante. Foram utilizados conectores para dar sentido ao DSC sem que isso alterasse a estrutura da frase elaborada ${ }^{14}$.

O estudo atendeu às exigências éticas presentes na Resolução no 466/2012 do Conselho Nacional de Saúde, aprovada pelo Comitê de Ética em Pesquisa da Universidade Federal do Triângulo Mineiro, em 2015, sob o Certificado de Apresentação para Apreciação Ética CAAE: 1 67860917.6.0000.5154, por meio do parecer número: 2.134.887.

\section{RESULTADOS}

Participaram 35 graduandos de enfermagem, dos quais: $80 \%$ do sexo feminino e $20 \%$ do sexo masculino; com média de idade de 24 anos; $54,28 \%$ do décimo período e $45,72 \%$ do nono período; $77,14 \%$ solteiros e $22,66 \%$ casados.

Emergiram quatro ICs e seus respectivos DSCs: Importância da disciplina de Educação em Saúde para o desenvolvimento acadêmico; Práticas da disciplina de Educação em Saúde pelo olhar dos discentes; Disciplina de Educação em Saúde como estratégia de ensino para população; e, Visão crítica do aprender Educação em Saúde.

\section{Importância da disciplina de Educação em Saúde para o desenvolvimento acadêmico}

DSC1. Para mim a disciplina de Educação em Saúde serviu mais para abrir os meus horizontes. Me mostrou novas maneiras de trabalhar com a população, principalmente na atenção primária. Foi importante para eu elaborar, traçar metas das atividades de acordo com as necessidades da população. Eu pude propor temas educacionais para o paciente no hospital e na comunidade. Me fez entender a necessidade de se educar a população. Consegui mostrar para os usuários as boas práticas de saúde. Eu pude desenvolver atividades com a finalidade de evitar alguns agravos 
na saúde. Eu aprendi a reconhecer as necessidades da população. Me auxiliou enquanto graduando a melhorar as práticas de saúde das pessoas. Com as ações de educação em saúde pude empoderar a comunidade. Penso que colaborei na partilha de conhecimentos sobre boas práticas de saúde e facilitei o diálogo entre os profissionais e a comunidade.

\section{Práticas da disciplina de Educação em Saúde pelo olhar dos discentes}

DSC2. Pude realizar de ações, de promoção e prevenção de saúde afim de conscientizar a população. Para mim foi aquele momento que tive contato com usuário do serviço de saúde e ensinei ele a se cuidar, melhorar sua condição de saúde, sua sobrevida, melhorar seu cotidiano. Pude desenvolver atividades com a população. Passei informações e orientações para os usuários dos serviços de saúde. Fiz com que a comunidade se interessasse pelo assunto discutido. Pude aprender como abordar a população de forma correta. Aprendi a verificar sempre se as pessoas estavam entendendo o que orientei e ensinei. Para mim essa disciplina fez eu aprender que essa é uma função muito grande da enfermagem que lida com paciente.

\section{Disciplina de Educação em Saúde como estratégia de ensino para população}

DSC3. São estratégias de como ensinar determinada população, com a finalidade de promover a saúde e na prevenção de doenças. Para mim foram as maneiras e as formas de como levar o conhecimento, trocar informações sobre saúde para a pessoas. Eu tive uma nova visão sobre algum tema educativo da melhor forma possível. Pude fazer com que a comunidade aprendesse sobre um assunto e que fossem multiplicadores na comunidade das informações aprendidas. Consegui aprender estratégias para conscientizar a população atendida por mim. Aprendi técnicas e estratégias para ensinar as pessoas terem o interesse pelo autocuidado. Aprendi que os usuários sejam responsáveis pela melhoria de sua condição de saúde. Aprendi que a disciplina de educação em saúde é estratégia segura para promover melhoria das condições vulneráveis de vida das pessoas quanto os agravos e peculiaridades que é causado na sua ausência.

\section{Visão crítica do aprender Educação em Saúde}

DSC4. Que a gente tenha mais incentivo durante a graduação. Para mim a disciplina poderia ter tido uma carga horária maior. Eu acho poderia ter tido mais atividades práticas, e ter a participação de outros professores. Deveria ser uma disciplina de mais integralizada. Eu gostaria de ter tido aulas mais dinâmicas com enfoque mais prático. Para mim a disciplina ficou presa demais na teoria. Eu acho que a parte prática poderia ter sido maior. Para mim poderia ter sido mais direcionada para os agravos de saúde que estão em evidência. Eu acho que os alunos deveriam ser mais ativos e interessados durante a disciplina. Para mim os alunos teriam que estar motivados desde o início e darem a devida importância à educação em saúde. Descobri a responsabilidade do enfermeiro frente ao cuidado com a população.

\section{DISCUSSÃO}

A disciplina demonstrou trazer consigo inúmeros benefícios para a formação do acadêmico de maneira efetiva e de qualidade de todos os aspectos que envolvem esse processo, com base nas DCNs do Curso de Enfermagem.

O enfermeiro deve considerar a ES como potente tecnologia em saúde que possibilita o encontro entre profissional e paciente representando espaço promotor de troca e aprendizado mútuo. Para tal, é necessário desenvolver competências e habilidades para atuar em diversas situações, dentre elas, reconhecer problemas em diferentes instâncias e particularidades da população a qual ele atende. Diante disso, propor soluções, sejam em níveis de prevenção, promoção da saúde ou reabilitação, para o indivíduo, família ou comunidade ${ }^{11}$. A ES representa uma ferramenta relevante para promover cuidado de qualidade de forma eficiente e eficaz, privilegiando o desenvolvimento para o autocuidado15.

Os graduandos apontaram a pertinência e o subsídio que a disciplina traz para a prática profissional. Trabalhos que na formação de enfermeiros para as ações de ES, a direção deve ser: baseado nos problemas e conhecimentos prévios de cada indivíduo, com vistas a transformação na dinâmica organizacional, mediante a proposta de problematização do processo de trabalho ${ }^{16,17}$. Estudos mostraram que acadêmicos de enfermagem que participaram de atividades educativas em saúde mostraram competência, imprescindível para atuação do enfermeiro, seja na pesquisa, no ensino, na extensão ou assistência ${ }^{18-20}$.

0 papel pedagógico do enfermeiro deve ser realizado de forma contínua, como uma parte natural da relação enfermeiro/cliente/paciente com habilidades e consciência pedagógica, bem como a capacidade de estar atento às necessidades dos clientes e pacientes, com o intuito de compreender e motivá-los para mudanças no estilo de vida ${ }^{16}$. Também, apesar da importância 
de se documentar as atividades de ES, na prática não se verifica isto, notadamente na enfermagem ${ }^{15-17}$.

O cuidado em saúde está inserido no âmbito da ES e em ações humanizadas, uma vez que constitui prática social que abrange processos capazes de desenvolver reflexão e consciência crítica dos envolvidos. A ES deve ter como base a comunicação por meio do diálogo entre as pessoas em um cenário plural promovendo o protagonismo autônomo de sua própria história, na perspectiva de mudanças significativas para melhoria da sua condição de vida ${ }^{20}$.

Neste sentido, as atividades de ES constituem aporte decisivo na formação do graduando, uma vez que ampliam referenciais que possibilitam contato com grandes questões contemporâneas e com a realidade social, o que faz dessa experiência termos de aprendizagem o compromisso ético e solidário com a sociedade ${ }^{4,6}$. Nesta perspectiva, oportuno ressaltar que a ES deve se fundamentar no olhar ampliado sobre a saúde, transcendendo ações vinculadas somente a condutas prescritivas e verticalizadas.

A preparação do graduando para realizar as atividades de ES tem sido um desafio, pois ao adotar estratégias dialógicas, foram observadas mudanças de comportamento dos participantes e de todos os envolvidos no processo. Os alunos podem então, atuar como facilitadores de novas possibilidades acerca das necessidades da população assistida, e das práticas adotadas para o cuidado em saúde ${ }^{19}$.

Foi possível identificar a importância da ES como estratégia de cuidado, visto que o educar em saúde é um mecanismo capaz de proporcionar transformações nas vidas e nos ambientes das pessoas que cuidamos. A ES exige pluralidade de percepções para a construção e ressignificação de conceitos e práticas. A ES busca na atuação da enfermagem a conscientização do indivíduo em relação a sua condição de saúde e, consequentemente, a criação de novos hábitos e comportamentos, favorecendo, assim, resultados positivos para a vida das pessoas e da coletividade 20 .

É necessário que ações educativas em saúde se tornem uma realidade nesse contexto, propiciando a criação de um cuidado integral, além da correlação entre assistência, educação e gestão, favorecendo a melhoria das práticas em saúde ${ }^{17}$. Dessa maneira, permite a criação de vínculo entre usuários e profissionais, fortalecendo o sistema, por meio da combinação e valorização de diversos saberes, contando com o protagonismo do paciente em todo o processo de mudança ${ }^{21,22}$.

A prática de ES pelo acadêmico de enfermagem deve ser uma experiência vivida ainda na formação, antecipada por conteúdos teóricos que o fundem $22-23$, o que permitirá ao futuro profissional implementar a ES como ferramenta de trabalho e mudança de paradigmas.

\section{CONCLUSÃO}

Pelos discursos, foi verificado que a disciplina de ES no contexto de formação do acadêmico de enfermagem, pode qualificar sua atuação, mostrando-se como ferramenta de trabalho e estratégia de promoção da saúde e prevenção de agravos.

É considerada como limitação do estudo o fato de o mesmo ter sido realizado em um único curso de graduação em enfermagem, com um número limitado de participantes, impossibilitando deste modo generalizações, assim outros estudos são necessários para que se conheçam outros contextos com o intuito de aprofundar o conhecimento produzido acerca da temática. Por sua vez, a pesquisa em questão traz a importância da ES enquanto disciplina na graduação em enfermagem, bem como, reforça a vivencia do aluno ainda em formação. 


\section{REFERÊNCIAS}

1. Vieira SL, Silva GTR, Silva RMO, Amestoy SC. Diálogo e ensino-aprendizagem na formação técnica em saúde. Trab Educ Saúde [Internet]. 2020 [citado em 20 mar 2021]; 18(Sup1):1-25. DOI: https://doi.org/10.1590/1981-7746-sol00253

2. Paulino VCP, Silva LA, Prado MA, Barbosa MA, Porto CC. Training and skills for teaching in nursing undergraduate courses. J Health NPEPS [Internet]. 2017 [citado em 27 abr 2020]; 2(1):272-84. DOI: https://doi.org/10.21675/2357-707X.2019.v10.n1.1954

3. Camila APLA, Fernandes DR, Amorim FCM, Veras JMMF, Oliveira ADS, Carvalho HEF, et al. 0 enfermeiro docente e o diabetes mellitus gestacional: o olhar sobre a formação. Enferm Foco [Internet]. 2019 [citado em 26 abr 2020]; 10(1):111-6. DOI: https://doi.org/10.21675/2357707X.2019.v10.n1.1954

4. Falkenberg MB, Benites MTPL, Moraes EP, Souza EM. Educação em saúde e educação na saúde: conceitos e implicações para a saúde coletiva. Ciênc Saúde Colet. [Internet]. 2014 [citado em 26 abr 2020]; 19(3):847-52. DOI: http://dx.doi.org/10.1590/1413-81232014193.01572013

5. Azevedo PRA, Sousa MM, Sousa NF, Oliveira SHS. Health education shares in the context of chronic diseases: integrative review. Rev Pesqui (Univ Fed Estado Rio J, Online) [Internet]. 2018 [citado em 20 abr 2020]; 10(1):260-7. DOI: http://dx.doi.org/10.9789/2175-5361.2018.v10i1.260-267

6. Mello AL, Brito LJS, Terra MG, Camelo SH. Estratégia organizacional para o desenvolvimento de competências de enfermeiros: possibilidades de educação permanente em saúde. Esc Anna Nery Rev Enferm. [Internet]. 2018 [citado em 02 mar 2020]; 22(1):1-10. DOI: https://doi.org/10.1590/2177-9465-ean-2017-0192

7. Mallmann DG, Galindo NNM, Sousa JC, Vasconcelos EMR. Educação em saúde como principal alternativa para promover a saúde do idoso. Ciênc Saúde Colet. [Internet]. 2015 [citado em 25 abr 2020]; 20(6):1763-72. DOI: https://doi.org/10.1590/1413-81232015206.02382014

8. Figueira AB, Amestoy SC, Cecagno D, Tristão FS, Trindade LL, Correa VA. Visão do enfermeiro frente à prática da educação em saúde no ambiente hospitalar. Cogitare Enferm. [Internet]. 2013 [citado em 30 abr 2020]; 18(2):3010-6. DOI: http://dx.doi.org/10.5380/ce.v18i2.32580

9. Pennafort VPS, Silva ANS, Queiroz MVO. Percepções de enfermeiras acerca da prática educativa no cuidado hospitalar a crianças com diabetes. Rev Gaúch Enferm. [Internet]. 2014 [citado em 30 abr 2020]; 35(3):130-6. DOI: https://doi.org/10.1590/1983-1447.2014.03.43313

10. Damiance PRM, Tonete VLP, Daibem DML, Ferreira MLSM, Bastos JRM. Formação para o SUS: uma análise sobre as concepções e práticas pedagógicas em saúde coletiva. Trab Educ Saúde [Internet]. 2016 [citado em 30 abr 2020]; 14(3)699-721. DOI: http://dx.doi.org/10.1590/19817746-sol00014

11. Ministério da Educação (Brasil), Conselho Nacional de Educação (Brasil). Parecer CNE/CES no 1.133 de 7 de outubro de 2001. [Dispõe sobre as] Diretrizes Curriculares Nacionais dos cursos de graduação de Enfermagem, Medicina e Nutrição [Internet]. Brasília, DF, 07 out 2001 [citado em 12 ago 2021]. Disponível em: http://portal.mec.gov.br/dmdocuments/ces1133.pdf

12. Magnago C, Pierantoni CR. A formação de enfermeiros e sua aproximação com os pressupostos das Diretrizes Curriculares Nacionais e da Atenção Básica. Ciênc Saúde Colet. [Internet]. 2020 [citado em 30 abr 2020]; 25(1):15-24. DOI: https://doi.org/I: 10.1590/141381232020251.28372019

13. Moraes BA, Costa NMSC. Understanding the curriculum the light of training guiding health in Brazil. Rev Esc Enferm USP [Internet]. 2016 [citado em 26 abr 2020]; 50(Esp):9-16. DOI: https://doi.org/10.1590/S0080-623420160000300002

14. Lefevre, F, Lefevre AMC. Discourse of the collective subject: social representations and communication interventions. Texto \& Contexto Enferm. [Internet]. 2014 [citado em 09 abr 2020]; 23(2):502-7. DOI: http://dx.doi.org/10.1590/0104-07072014000000014

15. Mota MS, Gomes GC, Petuco VM. Repercussions in the living process of people with stomas. Texto \& Contexto Enferm. [Internet]. 2016 [citado em 30 abr 2020]; 25(1):e1260014. DOI: https://doi.org/10.1590/0104-070720160001260014 
16. Halse KME, Fonn M. Health education and the pedagogical role of the nurse: nursing students learning in the clinical setting. J Nurs Educ Pract. [Internet]. 2014 [citado em 20 mar 2021]; 4(3):307. DOI: https://doi.org/10.5430/jnep.v4n3p30

17. Smith EMT. Junior baccalaureate nursing students: factors that predict success. J Nurs Educ Pract. [Internet]. 2020 [citado em 20 mar 2021]; 10(12):1-6. DOI: https://doi.org/10.5430/jnep.v10n12p1

18. Jesus MEF, Silva ABBF, Ramos JLC, Porcino C, Evangelista RP. Educação em saúde: concepções de discentes da graduação em enfermagem. Braz Appl Sci Rev. [Internet]. 2019 [citado em $30 \mathrm{abr}$ 2020]; 3(5):2263-75. DOI: https://doi.org/10.34115/basrv3n5-028

19. Silva PLN, Almeida SG, Martins AG, Gamba MA, Alves ECS, Silva JRF. Práticas educativas sobre violência contra a mulher na formação de universitários. Rev Bioét. [Internet]. 2016 [citado em 14 abr 2020]; 24(2):276-85. DOI: https://doi.org/10.1590/1983-80422016242128

20. Barbosa AS, Andrade GCL, Pereira CO, Falcão IV. A interdisciplinaridade vivenciada em um grupo de idosos de uma unidade de saúde da família do Recife. Rev APS [Internet]. 2016 [citado em 30 abr 2020]; 19(2):315-20. Disponível https://periodicos.ufjf.br/index.php/aps/article/view/15414

21. Costa AMS, Reis DA, Rocha TDP, Gomes YS, Mata LM. Educação em saúde em uma escola infantil do interior do Amazonas: relato de experiência. REVISA [Internet]. 2016 [citado em 25 abr 2020]; 9(1):125-32. DOI: https://doi.org/10.36239/revisa.v9.n1.p125a132

22. Mello AL, Brito LJS, Terra MG, Camelo SH. Organizational strategy for the development of nurses' competences: possibilities of Continuing Education in Health. Esc Anna Nery Rev Enferm. [Internet]. 2018 [citado em 25 abr 2020]; 22(1):20170192. DOI: https://doi.org/10.1590/2177-9465-ean2017-0192

Editora Associada: Vania Del Arco Paschoal

\section{CONTRIBUIÇÕES}

Michele Carla Gonçalves participou da concepção, coleta e análise dos dados e redação. Nylze Helena Guillarducci Rocha, Maria Paula Custódio Silva e Jesislei Bonolo do Amaral Rocha, colaboraram na redação e revisão. Bethania Ferreira Goulart contribuiu na revisão. Divanice Contim participou da concepção, coleta e análise dos dados, redação e revisão.

\section{Como citar este artigo (Vancouver)}

Gonçalves MC, Rocha NHG, Silva MPC, Goulart BF, Rocha JBA, Contim D. Educação em saúde no ensino de graduação em enfermagem: percepção dos acadêmicos. REFACS [Internet]. 2021 [citado em inserir dia, mês e ano de acesso]; 9(4):946-52. Disponível em: inserir link de acesso. DOI: inserir link do DOI.

\section{Como citar este artigo (ABNT)}

GONÇALVES, M. C.; ROCHA, N. H. G.; SILVA, M. P. C.; GOULART, B. F.; ROCHA, J. B. A.; CONTIM, D. Educação em saúde no ensino de graduação em enfermagem: percepção dos acadêmicos. REFACS, Uberaba, MG, v. 9, n. 4, p. 946-52, 2021. Disponível em: inserir link de acesso. Acesso em: inserir dia, mês e ano de acesso. DOI: inserir link do DOI.

\section{Como citar este artigo (APA)}

Gonçalves, M.C., Rocha, N.H.G., Silva, M.P.C., Goulart, B.F., Rocha, J.B.A., \& Contim, D. (2021). Educação em saúde no ensino de graduação em enfermagem: percepção dos acadêmicos. REFACS, 9(4), 94652. Recuperado em: inserir dia, mês e ano de acesso de inserir link de acesso. DOI: inserir link do DOI. 